
Not for reproduction, distribution or commercial use.

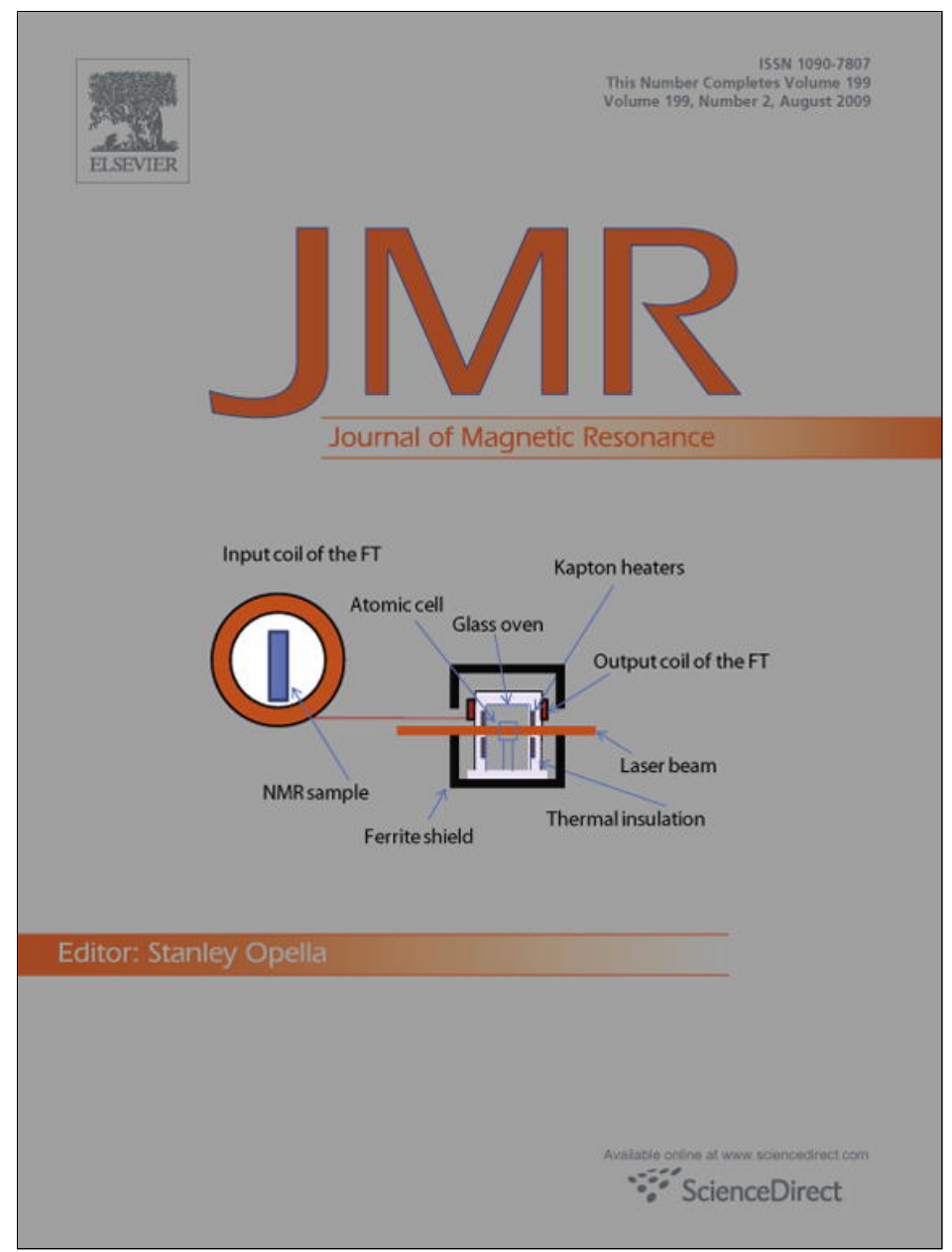

This article appeared in a journal published by Elsevier. The attached copy is furnished to the author for internal non-commercial research and education use, including for instruction at the authors institution and sharing with colleagues.

Other uses, including reproduction and distribution, or selling or licensing copies, or posting to personal, institutional or third party websites are prohibited.

In most cases authors are permitted to post their version of the article (e.g. in Word or Tex form) to their personal website or institutional repository. Authors requiring further information regarding Elsevier's archiving and manuscript policies are encouraged to visit:

http://www.elsevier.com/copyright 


\title{
Communication
}

\section{Low-power decoupling at high spinning frequencies in high static fields}

\author{
Markus Weingarth, Geoffrey Bodenhausen, Piotr Tekely* \\ Département de Chimie, associé au CNRS, Ecole Normale Supérieure, 24 rue Lhomond, 75231 Paris Cedex 05, France
}

\section{A R T I C L E I N F O}

\section{Article history:}

Received 17 March 2009

Revised 23 April 2009

Available online 3 May 2009

\section{Keywords:}

Low-power heteronuclear decoupling

Fast MAS

\begin{abstract}
A B S T R A C T
We demonstrate that heteronuclear decoupling using a Phase-Inverted Supercycled Sequence for Attenuation of Rotary ResOnance (PISSARRO) is very efficient at high spinning frequencies $\left(v_{\text {rot }}=60 \mathrm{kHz}\right)$ and high magnetic fields ( $900 \mathrm{MHz}$ for protons at $21 \mathrm{~T}$ ) even with moderate radio-frequency decoupling amplitudes $\left(v_{1}{ }^{I}=15 \mathrm{kHz}\right)$, despite the wide range of isotropic chemical shifts of the protons and the increased effect of their chemical shift anisotropy.
\end{abstract}

(c) 2009 Elsevier Inc. All rights reserved.

\section{Introduction}

Recently, a novel heteronuclear decoupling scheme dubbed PISSARRO (Phase-Inverted Supercycled Sequence for Attenuation of Rotary ResOnance) [1] was demonstrated to be efficient over a wide range of amplitudes $v_{1}{ }^{I}$ of the radio-frequency $(r f)$ field applied to the protons $I$. Initial trials were conducted at moderate spinning frequencies $(30 \mathrm{kHz})$ and medium static fields $(400 \mathrm{MHz}$ for protons at $9.4 \mathrm{~T}$ ). Under these conditions, PISSARRO decoupling proved to be more effective in quenching rotary resonance effects than established methods such as XiX [2,3], TPPM [4] or SPINAL-64 [5]. In particular, these conventional decoupling methods suffer from destructive interference due to rotary resonance effects, i.e., when $v_{1}{ }^{I} \approx n v_{\text {rot }}$ with $n=1,2,3 \ldots$. The interference of spinning and decoupling leads to undesirable line-broadening over wide ranges of $r f$ amplitudes $v_{1}{ }^{I}$, which is particularly annoying at high spinning frequencies $v_{\text {rot }}>30 \mathrm{kHz}$. For $\mathrm{CW}$ decoupling with $v_{\text {rot }}=40 \mathrm{kHz}$, rotary resonance effects broaden the lines of $\mathrm{CH}$ and $\mathrm{CH}_{2}$ groups unless $v_{1}{ }^{I}>200 \mathrm{kHz}$ [6] while for $v_{\text {rot }}=70 \mathrm{kHz}$, such interference effects only disappear if $v_{1}{ }^{I}>300 \mathrm{kHz}$ [7]. Thus it would seem that conventional decoupling methods would necessitate the use of ever increasing $r f$ amplitudes with increasing spinning frequencies. Fortunately, it has been shown recently that it is also possible to use decoupling methods such as CW, XiX, and TPPM with much lower $r f$ amplitudes $v_{1}{ }^{I}<<v_{\text {rot }}$, provided that $v_{\text {rot }}>40 \mathrm{kHz}[6,8,9]$. The efficiency of decoupling with such low $r f$ amplitudes is comparable to what can be achieved with high $r f$ amplitudes $v_{1}{ }^{I} \gg v_{\text {rot }}$. This Communication shows that at high spinning frequencies $\left(v_{\text {rot }}=60 \mathrm{kHz}\right)$ and high static fields (900 MHz for protons at $21 \mathrm{~T}$ ), PISSARRO decoupling remains

\footnotetext{
* Corresponding author. Fax: +33 144323344

E-mail address: Piotr.Tekely@ens.fr (P. Tekely).
}

remarkably efficient at commonly used $r f$ amplitudes $(\sim 100 \mathrm{kHz})$. Even with a much lower $r f$ amplitude $v_{1}{ }^{I}=15 \mathrm{kHz}$, PISSARRO performs just as well as high-power decoupling for $\mathrm{CH}_{3}$ and $\mathrm{CH}$ signals. For the more challenging $\mathrm{CH}_{2}$ groups, which have strong dipolar proton-proton couplings, low-amplitude PISSARRO with $v_{1}{ }^{I}=15 \mathrm{kHz}$ leads to a mere $20 \%$ loss in peak height compared to $v_{1}{ }^{I}=150 \mathrm{kHz}$. This permits a dramatic reduction of the $\mathrm{rf}$ power dissipation in the sample, which is vital for heat-sensitive samples such as hydrated proteins with high salt content.

\section{Experimental}

The PISSARRO sequence is composed of $N$ pulse pairs $\left(\tau_{\mathrm{p}}\right)_{\mathrm{x}}\left(\tau_{\mathrm{p}}\right)_{-\mathrm{x}}$ followed by $N$ pairs $\left(\tau_{\mathrm{p}}\right)_{\mathrm{y}}\left(\tau_{\mathrm{p}}\right)_{-\mathrm{y}}$ that together form a block. We shall use $N=5$ throughout this work. The choice of $N=5$ yields optimal performance for $20<v_{\text {rot }}<60 \mathrm{kHz}$. Four such blocks are combined to form a supercycle [1]. There is only a single adjustable parameter, i.e., the pulse width $\tau_{\mathrm{p}}$, or, equivalently, the ratio $\tau_{\mathrm{p}} / \tau_{\text {rot }}$, where the rotor period is $\tau_{\text {rot }}=1 / \nu_{\text {rot }}$.

Polycrystalline powders of uniformly ${ }^{13} \mathrm{C},{ }^{15} \mathrm{~N}$ labelled L-alanine and ${ }^{13} \mathrm{C}^{\alpha}$ labelled L-glycine were used without further purification. All experiments were performed on a $900 \mathrm{MHz}$ Bruker Avance II spectrometer with a double resonance $\mathrm{CP} / \mathrm{MAS}$ probe using rotors with $1.3 \mathrm{~mm}$ outer diameter spinning at $v_{\text {rot }}=60 \mathrm{kHz}$. Ramped cross polarization (CP) [10] and a simple excitation by a $90^{\circ}$ pulse were used to record spectra of L-glycine and L-alanine, respectively. Numerical simulations of PISSARRO and CW decoupling were carried out with SPINEVOLUTION [11]. For L-alanine, a cluster comprising one ${ }^{13} \mathrm{C}^{\alpha}$ nucleus and six intramolecular protons with distances derived from the crystallographic structure as well as two additional protons from the neighborhood was considered in the simulations. The latter two protons were assumed to be on resonance, and the rotation of the methyl protons was assumed to be very fast. 


\section{Results and discussion}

\subsection{Quenching interference due to rotary resonance}

The performance of $\mathrm{CW}$ and PISSARRO decoupling at $v_{\text {rot }}=60 \mathrm{kHz}$ MAS is compared in Fig. 1 for $\mathrm{CH}$ and $\mathrm{CH}_{2}$ groups. In the regime $v_{1}{ }^{I}>>v_{\text {rot }}$, CW decoupling suffers from recoupling effects due to rotary resonance $v_{1}{ }^{I} \approx n v_{\text {rot }}(n=1$ and 2) [12] over a wide range of $r f$ amplitudes. With PISSARRO, these interference effects almost completely disappear for $\mathrm{CH}$ and $\mathrm{CH}_{3}$ groups (the latter are not shown in Fig. 1), even with moderate $r f$ amplitudes $v_{1}{ }^{I} \approx 100 \mathrm{kHz}$. Compared to earlier observations at $v_{\text {rot }}=30 \mathrm{kHz}$ [1], the efficiency of PISSARRO with $v_{\text {rot }}=60 \mathrm{kHz}$ is particularly satisfying for $\mathrm{CH}_{2}$ groups. The recommended ratios are $\tau_{\mathrm{p}} / \tau_{\text {rot }}=0.305$ for $v_{1}{ }^{I} \approx v_{\text {rot }}, \tau_{\mathrm{p}} / \tau_{\text {rot }}=0.197$ for $v_{1}{ }^{I} \approx 2 v_{\text {rot }}$, and $\tau_{\mathrm{p}} / \tau_{\text {rot }}=0.9$ or 1.1 for higher $r f$ amplitudes $v_{1}^{I}>2 v_{\text {rot. }}$. Half and full rotor periods should be avoided, i.e., $\tau_{\mathrm{p}} / \tau_{\text {rot }} \neq k / 2$ with integer $k$. These recommendations can be followed blindly, although an empirical optimization in the vicinity of the recommended ratios $\tau_{\mathrm{p}} / \tau_{\text {rot }}$ may provide a minor improvement in decoupling efficiency. In practice, the optimal ratio $\tau_{\mathrm{p}} / \tau_{\text {rot }}$ is nearly the same at $v_{\text {rot }}=60$ and $30 \mathrm{kHz}$, which obviously makes it easy to set up PISSARRO decoupling.
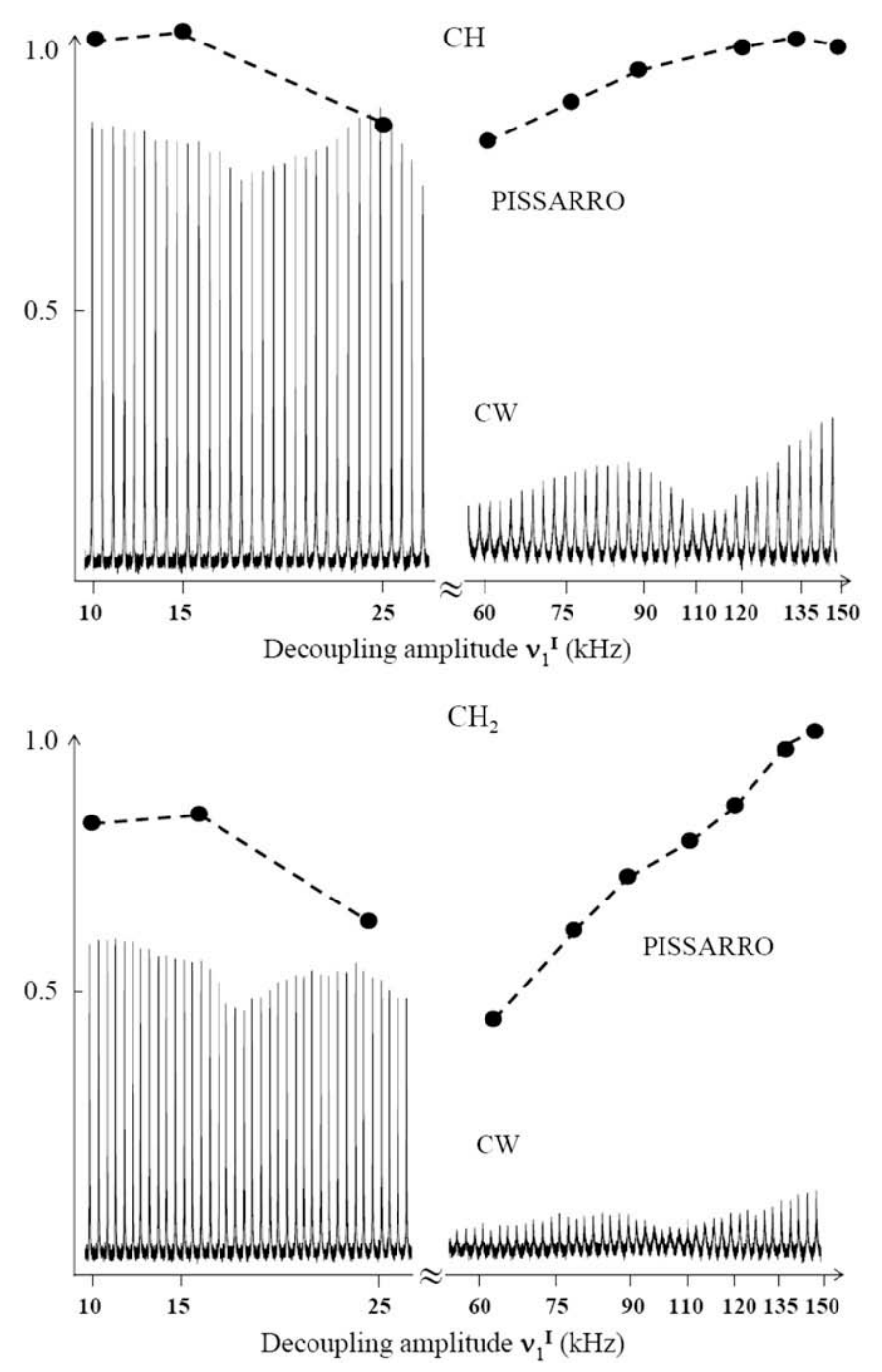

Fig. 1. Experimental intensities of the ${ }^{13} \mathrm{C}^{\alpha} \mathrm{H}$ peak in L-alanine (top) and the ${ }^{13} \mathrm{C}^{\alpha} \mathrm{H}_{2}$ resonance in L-glycine (below) as a function of the $r f$ decoupling amplitude $v_{1}{ }^{I}$. All spectra were obtained on a $900 \mathrm{MHz}$ spectrometer with $v_{\text {rot }}=60 \mathrm{kHz}$. The signals obtained by CW decoupling are represented by continuous lines, the peak heights obtained with PISSARRO decoupling by circles.
As in earlier work [1], we noted for $\mathrm{CH}$ groups a significant $50 \%$ drop of the performance of XiX decoupling near the $n=1$ rotary resonance condition, and a 30\% drop near $n=2$, in spite of careful optimization over a wide range of the pulse widths $\tau_{\mathrm{p}}$. For very high $r f$ amplitudes, XiX and PISSARRO offer the same decoupling performance. PISSARRO reaches the best efficiency at lower $r f$ amplitudes [1]. This is particularly important for $\mathrm{CH}_{2}$ groups with strong dipolar proton-proton couplings, where low-amplitude decoupling might not be sufficient in certain applications (vide infra).

\subsection{Low-amplitude decoupling}

As shown in Figs. 1 and 2, PISSARRO decoupling is also remarkably efficient in the low-amplitude regime. For $\mathrm{CH}_{3}$ and $\mathrm{CH}$ signals, despite a reduction of the $r f$ power by a factor of 100 , low-amplitude PISSARRO offered virtually the same decoupling performance as with the highest accessible amplitudes. On the other hand, the peak heights of $\mathrm{CH}_{2}$ groups observed with $v_{1}{ }^{I}=15 \mathrm{kHz}$ are only $\sim 20 \%$ lower. Such a loss might be acceptable for heat-sensitive biological samples. We found the optimal performance of low-power PISSARRO to be uniform for all resonances with $v_{1}{ }^{I}=15 \mathrm{kHz}$ when $v_{\text {rot }}=60 \mathrm{kHz}$, i.e., with $v_{1}^{I} / v_{\text {rot }}=1 / 4$. Nearly the same performance was observed with $v_{1}{ }^{I}=10 \mathrm{kHz}$. This stands in contrast to lowpower CW decoupling, where the optimal decoupling amplitude differs from resonance to resonance.

The middle columns of Fig. 2 show a comparison between peakshapes recorded with $v_{1}{ }^{I}=15$ and $25 \mathrm{kHz}$, the former corresponding to the optimal $r f$ strength for low-amplitude PISSARRO for all resonances, and the latter to the best $r f$ amplitude for $\mathrm{CH}$ resonances with $\mathrm{CW}$ decoupling. In all cases the lineshapes are affected by partially resolved ${ }^{13} \mathrm{C}-{ }^{13} \mathrm{C} \mathrm{J}$-couplings. This comparison reveals improvements of PISSARRO compared to CW decoupling of about $13 \%, 27 \%$, and $35 \%$ in the peak heights of the $\mathrm{CH}_{3}, \mathrm{CH}$ and $\mathrm{CH}_{2}$ resonance signals, respectively. For the $\mathrm{CH}$ resonance, the linewidths for $r f$ amplitudes of 150 and $15 \mathrm{kHz}$ are 114 and $119 \mathrm{~Hz}$, respectively, while $\mathrm{CW}$ decoupling at $25 \mathrm{kHz} r f$ amplitude leads to a linewidth of $125 \mathrm{~Hz}$. For the $\mathrm{CH}_{2}$ resonance, the corresponding linewidths are 118,128 , and $144 \mathrm{~Hz}$.

When using PISSARRO with low $r f$ fields, the pulse length $\tau_{\mathrm{p}}$ should be optimized so that the nutation angle $\beta=2 \pi v_{1}{ }^{I} \tau_{\mathrm{p}}$ is in the vicinity of a multiple of $2 \pi$ (Fig. 3 ). Furthermore, in analogy to high-amplitude PISSARRO, half and full rotor periods should be avoided, i.e., $\tau_{\mathrm{p}} / \tau_{\text {rot }} \neq k / 2$ with integer $k$. However, compared to high-amplitude PISSARRO, the dips near the conditions $\tau_{\mathrm{p}} / \tau_{\text {rot }}=k / 2$ are less pronounced, which makes the sequence more robust with respect to minor misadjustments and instrumental instabilities.

To appreciate in more detail to what extent CW and PISSARRO decoupling are sensitive to resonance offsets and CSA of the irradiated spins, we carried out numerical simulations of low-amplitude decoupling. In all simulations, the spinning frequency was $v_{\text {rot }}=60 \mathrm{kHz}$. In agreement with our experimental observations, the best performance of PISSARRO decoupling was found for $v_{1}{ }^{I}=15 \mathrm{kHz}$. The optimal pulse length $\tau_{\mathrm{p}}$ corresponds to a ratio $\tau_{\text {rot }} / \tau_{\mathrm{p}} \sim 3.86$, which is equivalent to a nutation angle $\beta \sim 349^{\circ}$. This is in good agreement with the experimental observation that the optimal nutation angles should be in the vicinity of multiples of $2 \pi$ For low-amplitude CW decoupling, the best performance was found for $v_{1}{ }^{I}=30 \mathrm{kHz}$, which roughly matches our experimental findings and relates to the so-called HORROR proton-proton recoupling condition $v_{1}{ }^{I}=n v_{\text {rot }}$ with $n=1 / 2$ [13]. Indeed, the resulting reintroduction of homonuclear couplings leads to a restoration of spin exchange in the proton network and improves the efficiency of CW decoupling in rotating solids [2,7].

Fig. 4a shows the height of the $\mathrm{C}^{\alpha}$ peak in L-alanine as a function of the offset of the proton carrier from the isotropic shift of the $\mathrm{H}^{\alpha}$ 

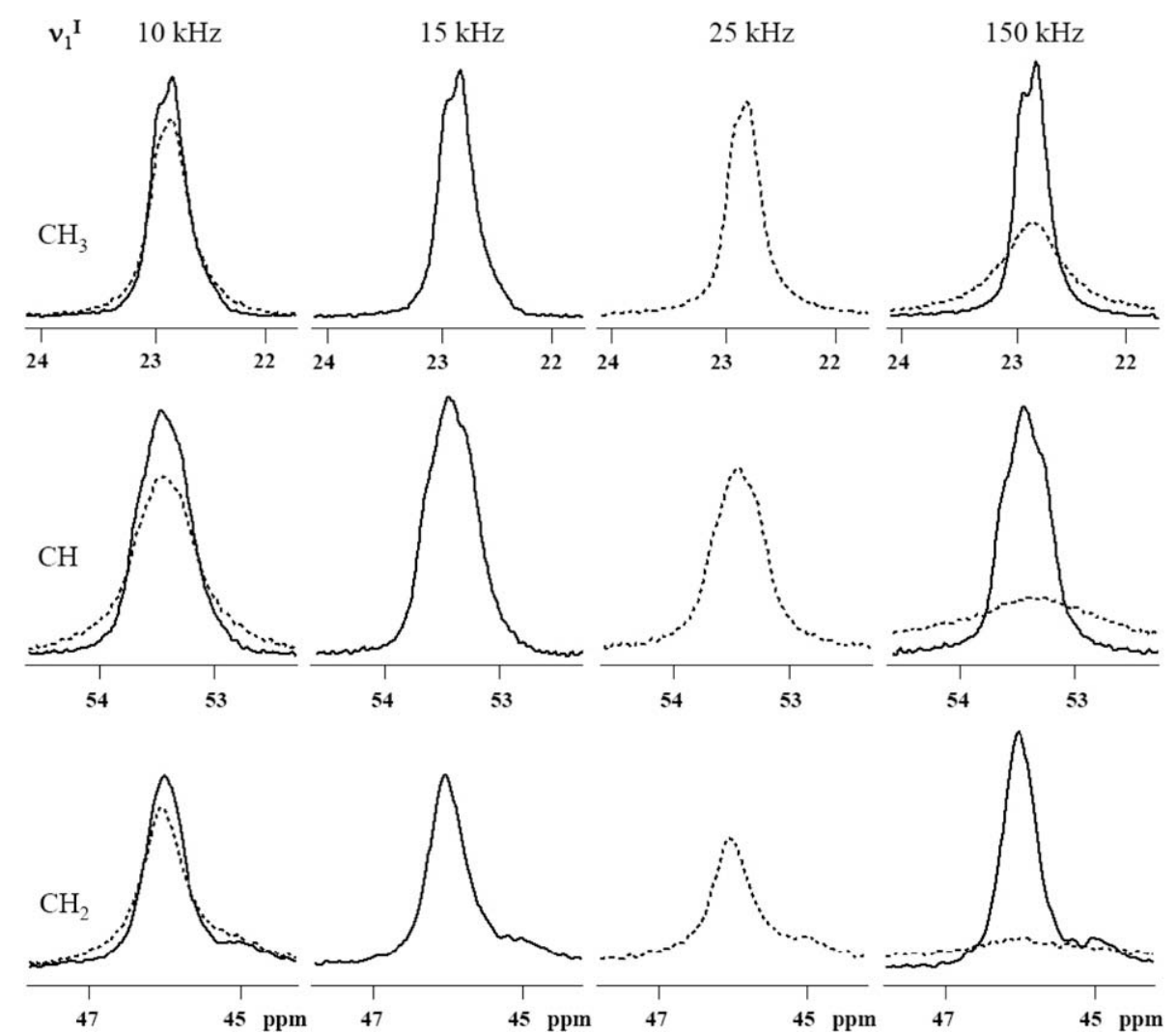

Fig. 2. Comparison of experimental peak-shapes of the ${ }^{13} \mathrm{C}^{\beta} \mathrm{H}_{3}$ and ${ }^{13} \mathrm{C}^{\alpha} \mathrm{H}$ peaks in L-alanine and the ${ }^{13} \mathrm{C}^{\alpha} \mathrm{H}_{2}$ peak in L-glycine at different $r f$ decoupling amplitudes $v_{1}{ }^{I}=10,15$, 25, and $150 \mathrm{kHz}$, using decoupling with PISSARRO (continuous lines) and CW (dotted lines) irradiation.

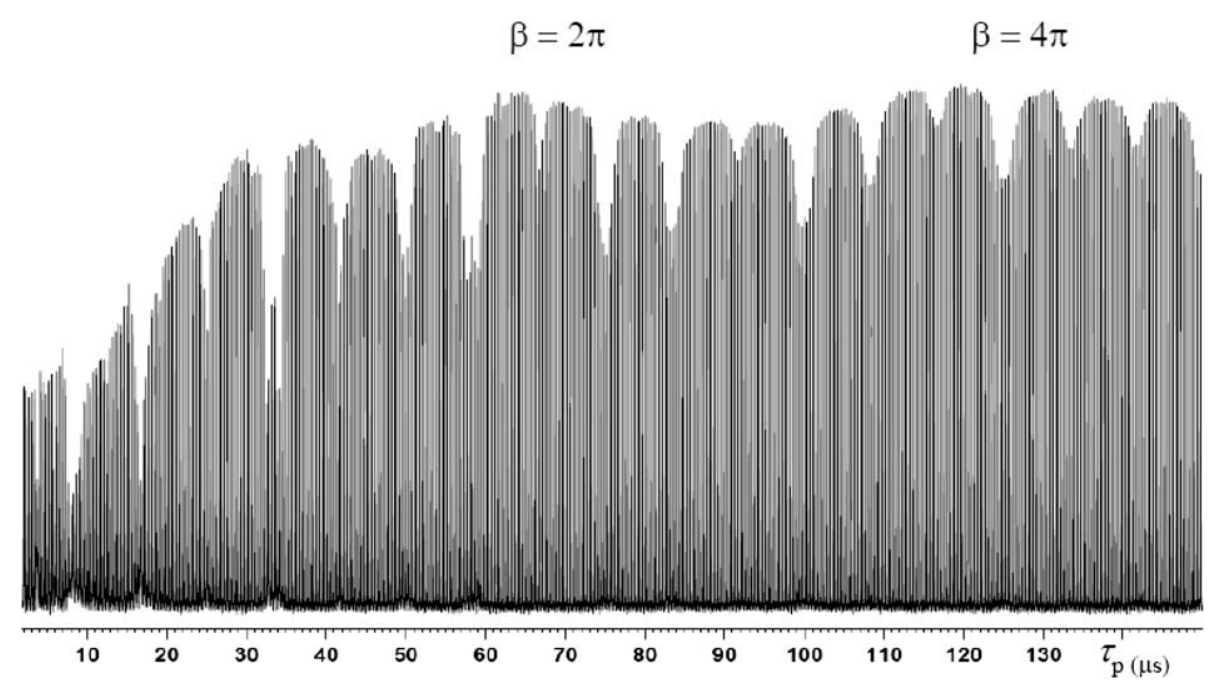

Fig. 3. Experimental ${ }^{13} \mathrm{C}^{\alpha} \mathrm{H}$ signals of L-alanine obtained with low-amplitude PISSARRO decoupling with $v_{1}{ }^{I}=15 \mathrm{kHz}$ as a function of the pulse width $\tau_{\mathrm{p}}$. Note the presence of dips due to undesired recoupling effects at multiples of half the rotor period, i.e., when $\tau_{\mathrm{p}} / \tau_{\text {rot }}=k / 2$ with integer $k$.

proton, simulated for two different static fields corresponding to 500 and $900 \mathrm{MHz}$. Fig. 4b shows simulations with and without CSA of the irradiated spin at $900 \mathrm{MHz}$. In contrast to PISSARRO, CW decoupling suffers from a pronounced offset dependence, even if the $r f$ amplitude is twice as high. This leads to line-broadening and hence to a loss of peak height at high magnetic fields. The presence of amino $\mathrm{H}^{\mathrm{N}}$ protons and $\mathrm{H}^{\beta}$ methyl protons as well as various residual homo- and heteronuclear dipolar couplings in L-alanine leads to the asymmetry in the responses of Fig. 4 . In fact, because of stronger couplings between the $\mathrm{C}^{\alpha}$ carbon and its directly at- tached $\mathrm{H}^{\alpha}$ proton with the neighboring $\mathrm{H}^{\mathrm{N}}$ protons than with the methyl protons, the broadening is less pronounced when the carrier frequency is displaced towards the $\mathrm{H}^{\mathrm{N}}$ resonance. To the best of our knowledge, the implications of the offsets of neighboring protons have not been considered so far in the context of heteronuclear decoupling. A different type of off-resonance decoupling has been reported in calcium formate [14], which contains only a single proton species. In this case, the improved off-resonance decoupling was ascribed to a partial cancellation of higher-order crossterms. 

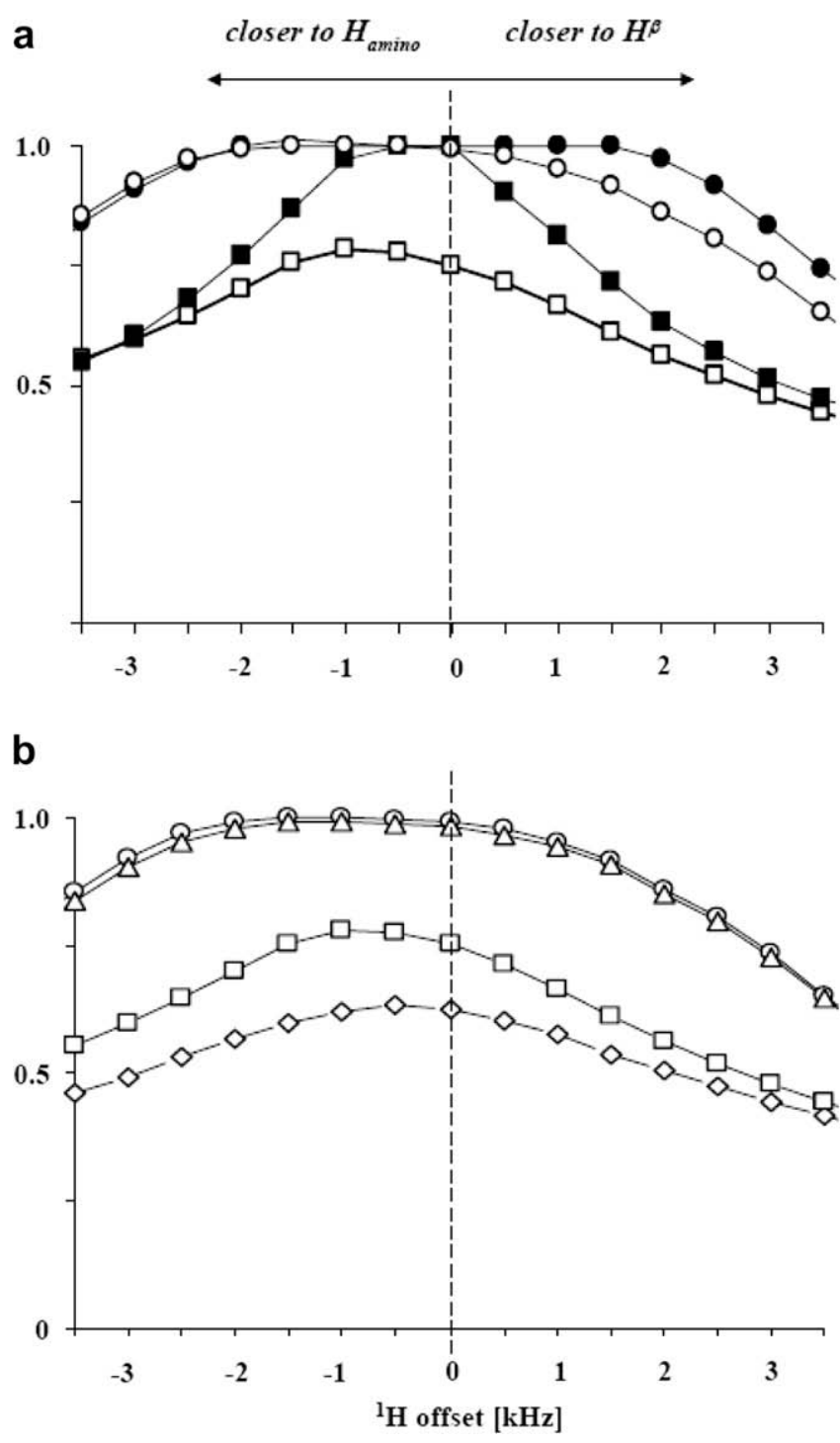

Fig. 4. (a) Numerical simulations of the ${ }^{13} \mathrm{C}^{\alpha} \mathrm{H}$ peak heights in L-alanine as a function of the carrier frequency of the proton decoupler: (squares) low-amplitude $\mathrm{CW}$ decoupling $\left(v_{1}^{I}=30 \mathrm{kHz}\right)$ and (circles) low-power PISSARRO decoupling $\left(v_{1}{ }^{I}=15 \mathrm{kHz}\right)$ at $v_{\text {rot }}=60 \mathrm{kHz}$. The filled and open symbols represent simulations for ${ }^{1} \mathrm{H}$ resonance frequencies of 500 and $900 \mathrm{MHz}$. (b) Simulations for lowamplitude CW decoupling (squares, lozenges) and low-power PISSARRO (circles, triangles) at $900 \mathrm{MHz}$, calculated without (squares, circles) and with (lozenges, triangles) a proton chemical shift anisotropy $\Delta \sigma=\sigma_{33}-\sigma_{\text {iso }}=5 \mathrm{ppm}(4.5 \mathrm{kHz}$ at $900 \mathrm{MHz}$ ). The peak heights were normalized with respect to the best performance of low-amplitude PISSARRO decoupling.

CW decoupling suffers from second-order recoupling effects due to cross-terms between the heteronuclear dipole-dipole and anisotropic chemical shielding interactions of the irradiated spins [15]. Indeed, as demonstrated in the simulations of Fig. 4b, lowamplitude CW decoupling is significantly affected by the CSA of the irradiated spins, while the efficiency of low-amplitude PISSAR-
RO decoupling remains virtually unchanged. This is yet another reason why PISSARRO decoupling should be preferred at very high static fields.

\section{Conclusions}

We have shown that the most important feature of PISSARRO decoupling, i.e., its ability to quench interference effects due to rotary resonance, improves at high spinning frequencies. Because of the compensation of resonance offsets and of the chemical shift anisotropy of the irradiated spins, low-amplitude PISSARRO decoupling is particularly efficient at fast spinning in very high static fields.

\section{Acknowledgments}

The Research Infrastructure Activity in the 6th framework Program of the EU (contract No. RII3-026145, EU-NMR) and the European Center for High Field NMR in Lyon are greatly acknowledged. We thank P.K. Madhu and Michal Leskes for help with SPINEVOLUTION simulations.

\section{References}

[1] M. Weingarth, P. Tekely, B. Bodenhausen, Efficient heteronuclear decoupling by quenching rotary resonance in solid-state NMR, Chem. Phys. Lett. 466 (2008) 247-251.

[2] P. Tekely, P. Palmas, D. Canet, Effect of proton spin exchange on the residual ${ }^{13} \mathrm{C}$ MAS NMR linewidths, phase-modulated irradiation for efficient heteronuclear decoupling in rapidly rotating solids, J. Magn. Reson. A 107 (1994) 129-133.

[3] A. Detken, E.H. Hardy, M. Ernst, B.H. Meier, Simple and efficient decoupling in magic-angle spinning solid-state NMR: the XiX scheme, Chem. Phys. Lett. 356 (2002) 298-304

[4] A.E. Bennett, C.M. Rienstra, M. Auger, K.V. Lakshli, R.G. Griffin, Heteronuclear decoupling in rotating solids, J. Chem. Phys. 103 (1995) 6951-6958.

[5] B.M. Fung, A.K. Khitrin, K. Ermolaev, An improved broadband decoupling sequence for liquid crystals and solids, J. Magn. Reson. 142 (2000) 97-101.

[6] M. Kotecha, N.P. Wickramasinghe, Y. Ishii, Efficient low-power heteronuclear decoupling in ${ }^{13} \mathrm{C}$ high-resolution solid-state NMR under fast magic angle spinning, Magn. Reson. Chem. 45 (2007) S221-S230.

[7] M. Ernst, A. Samoson, B.H. Meier, Decoupling and recoupling using continuouswave irradiation in magic-angle-spinning solid-state NMR: a unified description using bimodal Floquet theory, J. Chem. Phys. 123 (2005) (064102-1-10).

[8] M. Ernst, A. Samoson, B.H. Meier, Low-power decoupling in fast magic-angle spinning NMR, Chem. Phys. Lett. 348 (2001) 293-302.

[9] M. Ernst, A. Samoson, B.H. Meier, Low-power XiX decoupling in MAS NMR experiments, J. Magn. Reson. 163 (2003) 332-339.

[10] G. Metz, X.L. Wu, S.O. Smith, Ramped-amplitude cross-polarization in magicangle-spinning NMR, J. Magn. Reson. A 110 (1994) 219-227.

[11] M. Veshtort, R.G. Griffin, SPINEVOLUTION: a powerful tool for the simulation of solids and liquid state NMR experiments, J. Magn. Reson. 178 (2006) 248282.

[12] T.G. Oas, R.G. Griffin, M.H. Levitt, Rotary resonance recoupling of dipolar interactions in solid-state nuclear magnetic resonance, J. Chem. Phys. 89 (1988) 692-695.

[13] N.C. Nielsen, H. Bildsoe, H.J. Jakobsen, M.H. Levitt, Double-quantum homonuclear rotary resonance. Efficient dipolar recovery in magic-angle spinning nuclear magnetic resonance, J. Chem. Phys. 101 (1994) 1805-1812.

[14] M. Eden, M.H. Levitt, Pulse sequence symmetries in the nuclear magnetic resonance of spinning solids: application to heteronuclear decoupling, J. Chem. Phys. 111 (1999) 1511-1519.

[15] M. Ernst, S. Bush, A.C. Kolbert, A. Pines, Second-order recoupling of chemicalshielding and dipolar-coupling tensors under spin decoupling in solid-state NMR, J. Chem. Phys. 106 (1996) 3387-3397. 